\title{
The Architecture Analysis of Internet of Things
}

\author{
Nihong Wang and Wenjing $\mathrm{Wu}^{*}$ \\ Information and Computer Engineering College of Northeast Forestry University, \\ Heilongjiang Harbin 150040, China \\ hlg05wj@126.com, wangnh989@163.com
}

\begin{abstract}
The Internet of Things, as an emerging global Internet-based technical architecture, facilitates the exchange of goods and services in global supply chain networks. The phrase Internet of Things (IoT) heralds a vision of the future Internet where connecting physical things, from banknotes to bicycles, through a network will let them take an active part in the Internet, exchanging information about themselves and their surroundings. The IoT industry is still in an early stage of development, whose technologies, standards, product and the market still need to be improved, and whose related areas also need to be studied and discussed. This paper presents detailed analysis and comparison about a misunderstanding of the current awareness: IoT $=$ Internet + WSN, which enable people to understand IoT more thoroughly. The IoT is a network of connecting objects, whose architecture is different from that of the traditional network, which can not simply described by the use of layered network architecture. In this paper, based on the IoT itself, the three dimensions architecture is researched and found, which is the most suitable architecture of IoT.
\end{abstract}

Keywords: the Internet of Things, architecture analysis, wireless sensor network, the three dimensions architecture.

\section{Introduction}

In 1991, Mark Weiser described the vision of a future world under the name of Ubiquitous Computing. Since then, many details of the described vision have become reality: Our mobile phones are powerful multimedia systems, our cars computer systems on wheels, and our homes are turning into smart living environments. All these advances must be turned into products for very cost-sensitive world markets in shorter cycles than ever before [1].

The Internet of Things (IoT) is a network that combines all kinds of information sensing equipments with the internet. Its purpose is that all the items can be perceived and controlled remotely, and combined with the internet to form a more wisdom production and living systems. IoT describes a world where humans are surrounded by machines that communicate with each other and can allow people to interact with

\footnotetext{
* This work was supported by the foundation of the forestry nonprofit industry scientific research special project of the State Forestry Administration, namely "The monitoring technology based on the IoT and its application research in forestry."
} 
the digital world. To succeed in this vision, it is not only the people who need an understanding of this multi-device environment, but also the network needs a representation of "who" the user is [2]. Vividly speaking, with the help of IoT, the car will automatically alarm when a misuse occurs in driving; the briefcase will put the owner in mind of something that he forgets; the clothes will tell the color and temperature of their requirement to washing machine; the outfall will send out a warning when the pollution exceeds the normal level; the traffic lights will dynamically control according to the crossing situation of pedestrian...

The concept of IoT break the traditional ideas and start a new technology field. Physical infrastructure and IT infrastructure has been separated in tradition. There are the infrastructure construction, such as airports, buildings, etc. on the one hand, and the data center, such as personal computers, broadband, etc. on the other hand. These two aspects exist relatively independently. However, they are integrated into a unified infrastructure in the era of IoT. The Internet of Things as an emerging global Internetbased information architecture facilitating the exchange of goods and services is gradually developing [3]. It will bring a new revolution of world information industry after the computer, internet and mobile communication network.

\section{$2 \quad$ IoT $=$ Internet + WSN (Wireless Sensor Network)?}

In the research communities, IoT has been defined from various different perspectives. The reason of today apparent fuzziness around this term is a consequence of the name "Internet of Things" itself, which syntactically is composed of two terms. The first one pushes towards a network oriented vision of IoT, while the second one moves the focus on generic "objects" to be integrated into a common framework [4].The Internet of Things is an extension and expansion network based on the internet, whose users extend and expand to the information exchange and communications between any goods. It leads to the emerging saying: IoT $=$ Internet + WSN. To analyze the correctness of this statement, the differences between IoT, internet and WSN are compared below (in Table.1).

From the analysis and comparison of characteristics in table 1 , IoT $\neq$ Internet + WSN is obvious. There are two clear points here. Firstly, IoT may not necessarily use IP network. At least, the IP network that can only provide "best effort" transmission capacity is not inappropriate. The IoT has much higher requirement to its bearer network than the current internet. Secondly, especially small intelligent objects network, IoT requires the best use of lightweight communication protocol. So, complex protocol like TCP/IP can not be used in the smart little things network. By starting from the considerations above, IoT will be a different environment from the internet, but can not be a simple extension of current Internet.

Since the IoT is not only a mere extension of today's Internet, but rather a complex netting of independent but interoperable systems, implemented in a symbiosis with new services and different modes of communication, the traditional Internet Governance concepts are not anymore suitable to identically be applied [5]. We should speed up the process of information social, which lays a good foundation for the development of IoT. 
Table 1. Differences Between Internet, WSN and IoT

\begin{tabular}{|c|c|c|c|}
\hline Feature items & Internet & WSN & IoT \\
\hline Identify goods & Can not & Can & Must \\
\hline Perceive goods & Can not & Can & Must \\
\hline Nodes types & Active & Active & Active and passive \\
\hline Network coverage & Wide area & Local area & Wide area \\
\hline $\begin{array}{l}\text { The number of } \\
\text { network nodes }\end{array}$ & Unlimited & Limited & Unlimited \\
\hline $\begin{array}{l}\text { Networking } \\
\text { approaches }\end{array}$ & $\begin{array}{l}\text { Determine the } \\
\text { backbone, } \\
\text { flexible access }\end{array}$ & Self-organization & $\begin{array}{l}\text { Determine the } \\
\text { backbone, } \\
\text { Independent access }\end{array}$ \\
\hline Networking time & Unlimited & Unlimited & $\begin{array}{l}\text { Timing } \\
\text { synchronization }\end{array}$ \\
\hline $\begin{array}{l}\text { Networking data } \\
\text { processing }\end{array}$ & End nodes & $\begin{array}{l}\text { End nodes, } \\
\text { aggregation nodes }\end{array}$ & All nodes \\
\hline $\begin{array}{l}\text { Information } \\
\text { relevance }\end{array}$ & Unrelated & Related & Related \\
\hline $\begin{array}{l}\text { Application } \\
\text { relevance }\end{array}$ & Unrelated & Unrelated & Related \\
\hline $\begin{array}{l}\text { Items semantic } \\
\text { recognition }\end{array}$ & No & End nodes & $\begin{array}{l}\text { End nodes, } \\
\text { aggregation nodes }\end{array}$ \\
\hline $\begin{array}{l}\text { Items semantic } \\
\text { processing }\end{array}$ & No & End nodes optional & $\begin{array}{l}\text { End nods, } \\
\text { aggregation nodes }\end{array}$ \\
\hline $\begin{array}{l}\text { self feedback } \\
\text { control }\end{array}$ & No & End nodes optional & $\begin{array}{l}\text { Multi-stage self- } \\
\text { feedback }\end{array}$ \\
\hline
\end{tabular}

\section{The Three Dimensions Architecture of IoT}

The IoT has the potential to add a new dimension to this process by enabling communications with and among smart objects, thus leading to the vision of "anytime, anywhere, anymedia, anything" communications. To this purpose, we observe that the IoT should be considered as part of the overall Internet of the future, which is likely to be dramatically different from the Internet we use today [4]. The IoT is a network in which everything can communicate with each other. Its architecture that is different from the traditional network's, should not be simply described through layered network architecture.

The system of IoT itself is composed of three dimensions which contain information items, independent networks and intelligent applications. In Fig. 1, the main concepts, technologies and features are highlighted and classified with reference to the three dimensions of IoT. The diagram clearly depicts that IoT paradigm will lead to the convergence of the three main dimensions addressed of IoT [6]. Information items are those that can identify and perceive their own message; independent networks have the capabilities of self-configuration, self-healing, selfoptimizing, self-protection; intelligent applications mean the application with the 
capabilities of intelligent control and processing. The three dimensions of IoT (including the independent network) are that the traditional network system does not have, but that the network connected items must have. Otherwise, IoT will not be able to meet the application requirements.

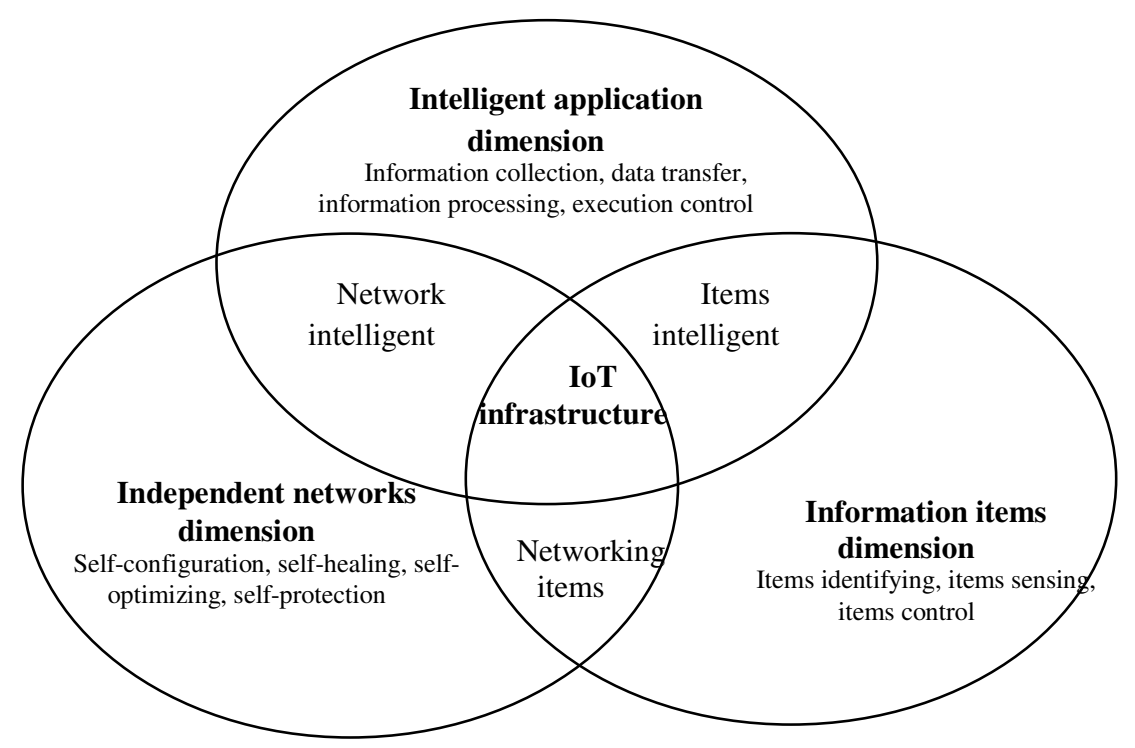

Fig. 1. Three dimensions architecture of IoT

The overlapping part of information items, independent networks and intelligent applications is the system of IoT with all the characteristics of IoT, which can be called the IoT infrastructure. Currently, the IoT system is not perfect but a total of general network system to connect items such as Intelligent Transportation System, Intelligent Electric Grid System, Smart City. The infrastructure of IoT here means that the support systems to serve the specific things, which can provide various services including the goods identification, location identification of space objects, features authentication of items' data and privacy protection in different application areas. It is the core of public Internet of Things.

The IoT needs to include items feature dimension, which is that the traditional networks don't have. Items connected to the IoT can be called information items, which have the basic features: with electronic identification, can transmit information. The networks constituting IoT need to connect a variety of items, which have the features of self-configuration and self-protection at least, and belong to a kind of independent network; the application of IoT generally related items which have the automatic collection, transmission and data processing, routine control automatically, and belongs to a kind of intelligent application.

Independent networks are an advanced form of today's networks, which will be simplified to a general network once treat with self-configuring, self-healing, selfoptimizing and self-protection, the network can be describe using the hierarchical 
model; if intelligence applications are entirely processed through a man-machine interactive interface, it will be reduced to an ordinary network application; if IoT no longer connect items directly but input the item information through the manmachine interactive interface, it will no longer need to identify the items and automatically transfer items information. In this way, the IoT will be simplified into a general network system, which can be described by the layered architecture of modern network. So, existing Internet architecture can be considered as a special case of the three dimensions architecture of IoT.

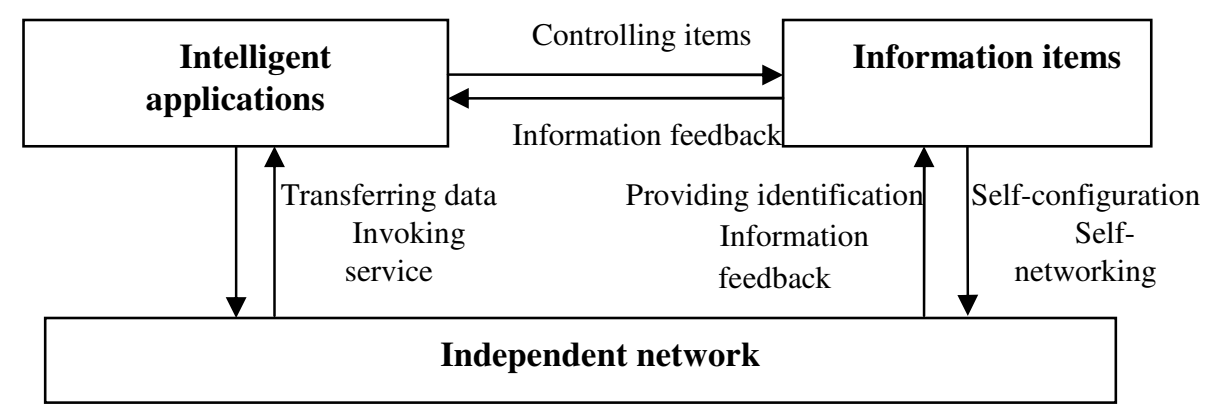

Fig. 2. The relationships of three Dimensions of IoT

The relationships between three dimensions of IoT are vividly showed in Fig.2, which make us understand the meaning of IoT intensively. The characteristic of IoT can be analyzed and evaluated, and network system can be determined that whether it is the IoT system by three visions architecture model of IoT. For example, if a network can only connect and perceive the goods but do not have the intelligent application, which does not belong to a complete IoT. Therefore, the sensor network does not belong to a complete IoT, which just has the features of independent network and information items.[7]

\section{Conclusion}

The IoT can be applied extensively in all walks of life such as automation and industrial manufacturing, logistics, business / process management, intelligent transportation, intelligent home, etc., which can be used widely in the agricultural fields. In terms of the different stages in agricultural production, the IoT can be used to improve its efficiency from the planting stage to harvesting stage. For example, in the preparation stage of cultivation, a lot of sensors are arranged in the greenhouse, which can analyze real-time soil information to choose the right crops. In the stages of planting and nurturing, the IoT can be used to collect temperature and humidity information, which provides high-efficient management to respond to environmental changes. Then in the harvest stage of crops, the IoT can be also used to gather the information of various properties in its transmission stage and use stage, back to the front, which can estimate accurately in the stage of harvest. The communication 
between objects is required, and will create notion value, thus will provide impetus and opportunities for the development of communication, which is the value of IoT.

The vision of the Internet of Things is in an embryonary state. Most of the elements of our world, our things do not have the digital intelligence that enables them to be aware of the existence of a virtual cyberworld, where they could be able to collaborate with literally billions of entities: other things, humans, computational processes, our own physical environment, and so on [8]. NIC foresees that "by 2025 Internet nodes may reside in everyday things - food packages, furniture, paper documents, and more". It highlights future opportunities that will arise, starting from the idea that "popular demand combined with technology advances could drive widespread diffusion of an Internet of Things (IoT) that could, like the present Internet, contribute invaluably to economic development". The possible threats deriving from a widespread adoption of such a technology are also stressed. Indeed, it is emphasized that "to the extent that everyday objects become information security risks, the IoT could distribute those risks far more widely than the Internet has to date".

We should provide effective legal guarantee, supporting policy for IoT by legal, administrative and economic means, so that IoT really become an open, secure, trustable network. Then, we can realize the integration of human social and physical system, and manage the production and life more meticulously and dynamically, and improve the utilization rate of resources and productivity, consequently, improve the relationship between man and nature.

\section{References}

1. Zuehlke, D.: SmartFactory-Towards a factory-of-things. Annual Reviews in Control 34, 129-138 (2010)

2. Sarma, A.C., Girão, J.: Identifies in the Future Internet of Things. Wireless Pers. Commun. 49, 353-363 (2009)

3. Weber, R.H.: Accountability in the Internet of Things. Computer Law \& Security Review 27, 133-138 (2011)

4. Atzori, L., Iera, A., Morabito, G.: The Internet of Things: A survey. Computer Networks, 119 (2010)

5. Weber, R.H.: Internet of things- Need for a new legal environment? Computer Law \& Security Review 25, 522-527 (2009)

6. Bandyopadhyay, D., Sen, J.: Internet of Things: Applications and Challenges in Technology and Standardization. Wireless Pers. Commun. 58, 49-69 (2011)

7. Shen, S.-B., Mao, Y.-Q., Fan, Q.-L., et al.: Conception Model and architecture of the Internet of Things. Journal of Nanjing University of Posts and Telecommunications (Natural Science) 30(4), 1-8 (2010)

8. Roman, R., Alcaraz, C., Lopez, J., et al.: Key management systems for sensor networks in the context of the Internet of Things. Computers and Electrical Engineering 37, 147-159 (2011) 